\title{
Construção e validação de um caso clínico para uso em simulação do exame físico do
}

\section{sistema respiratório}

\section{Construction and validation of a clinical case for use in simulation of the physical examination of}

\author{
the respiratory system
}

Construcción y validación de un caso clínico para utilizan en la simulación de la exploración física

\author{
del aparato respiratório
}

Recebido: 12/05/2021 | Revisado: 18/05/2021 | Aceito: 21/05/2021 | Publicado: 07/06/2021

Patrícia Costa dos Santos da Silva ORCID: https://orcid.org/0000-0001-9643-1865

Universidade Federal de Uberlândia, Brasil E-mail: patriciacosta@ufu.br

Nathalia Amado da Silva Medeiros ORCID: https://orcid.org/0000-0003-0929-5418

Universidade Federal de Uberlândia, Brasil E-mail: nath_eu@hotmail.com

Carolina de Freitas Cortes

ORCID: https://orcid.org/0000-0002-6904-3231

Universidade Federal de Uberlândia, Brasil

E-mail: caarolfcortes@gmail.com

Clesnan Mendes-Rodrigues

ORCID: https://orcid.org/0000-0002-8871-7422

Universidade Federal de Uberlândia, Brasil

E-mail: clesnan@hotmail.com

Kleber Gontijo de Deus

ORCID: https://orcid.org/0000-0003-0892-9968

Universidade Federal de Uberlândia, Brasil E-mail: klebergontijo@ufu.br

Luana Araújo Macedo Scalia

ORCID: https://orcid.org/0000-0003-1000-8738

Universidade Federal de Uberlândia, Brasil E-mail: luanascalia@ufu.br

Ricardo Gonçalves de Holanda ORCID: https://orcid.org/0000.0002.2347-4810 Universidade Federal de Uberlândia, Brasil E-mail: profricardoholanda@ufu.br

Caio César Gonçalves de Holanda ORCID: https://orcid.org/0000-0003-0339-8635 Universidade Federal de Uberlândia, Brasil

E-mail: caiocesarholanda@gmail.com

Suely Amorim de Araújo

ORCID: https://orcid.org/0000-0001-9234-166X Universidade Federal de Uberlândia, Brasil

E-mail: profasuelyamorim@ufu.br

\begin{abstract}
Resumo
Objetivo: Elaborar e validar um caso clínico para ser utilizado na simulação de uma situação realística do exame físico do sistema respiratório em um laboratório de práticas do Curso de Enfermagem. Métodos: Trata-se de estudo metodológico de construção e de validação de um caso clínico para ser utilizado em uma aula de simulação do exame físico do sistema respiratório. A caracterização da amostra de docentes e alunos que participaram na validação foi analisada de forma descritiva, sendo que os dados foram apresentados como média, mediana e desvio padrão O projeto foi aprovado pelo Comitê de Ética em Pesquisa da Universidade Federal de Uberlândia, sob o parecer $\mathrm{n}^{\circ}$ 1.864.940. Resultados: Os avaliadores foram predominantemente do sexo feminino nos dois grupos. A idade foi diferente nos dois grupos $(\mathrm{p}=0,008)$, sendo os docentes apresentaram idade mediana superior aos alunos (38 versus 23 anos). Quanto aos itens da validação do caso clínico, nenhum apresentou diferença significativa entre os dois grupos, docentes versus discentes $(\mathrm{p}>0,05)$. Todos os itens receberam avaliação mediana superior a 2,75 e média superior a 2,48. Conclusão: O caso clínico foi elaborado e validado pelos enfermeiros especialistas e pelos alunos do Curso de Graduação em Enfermagem.
\end{abstract}


Palavras-chave: Enfermagem; Simulação; Educação em enfermagem.

\begin{abstract}
Objective: To elaborate and validate a clinical case to be used in the simulation of a realistic situation of the physical examination of the respiratory system in a laboratory of nursing course practices. Methods: This is a methodological study of the construction and validation of a clinical case to be used in the video simulation of the physical examination of the respiratory system. The characterization of the sample of the teachers and students who participated in the validation was analyzed descriptively, and the data were presented as: mean, median and standard deviation. The project was approved by the Research Ethics Committee of the Federal University of Uberlândia under the opinion No. 1,864,940. Results: The evaluators were predominantly female in both groups. The age group was different in the two groups $(\mathrm{p}=0.008)$, and the teachers had a median age higher than the students (38 versus 23 years). Regarding the validation of the clinical case, there were no significant differences between the two groups, teachers versus students ( $p>0.05$ ). All items received a median score greater than 2.75 and an average score greater than 2.48. Conclusion: It is concluded that the clinical case was elaborated and validated by the specialist nurses and the undergraduate nursing students.
\end{abstract}

Keywords: Nursing; Simulation; Education in nursing.

\title{
Resumen
}

Objetivo: Elaborar y validar un caso clínico para ser utilizado en la simulación de una situación realista del examen físico del sistema respiratorio en un laboratorio de prácticas de cursos de enfermería. Métodos: Este es un estudio metodológico de la construcción y validación de un caso clínico que se utilizará en la simulación de video del examen físico del sistema respiratorio. La caracterización de la muestra de los docentes y estudiantes que participaron en la validación se analizó de forma descriptiva y los datos se presentaron como: media, mediana y desviación estándar. El proyecto fue aprobado por el Comité de Ética de Investigación de la Universidad Federal de Uberlândia bajo la opinión de $\mathrm{N}^{\mathrm{o}}$ 1.864.940. Resultados: los evaluadores fueron predominantemente mujeres en ambos grupos. El grupo de edad fue diferente en los dos grupos $(\mathrm{p}=0,008)$ y los maestros tenían una edad media mayor que los estudiantes (38 versus 23 años). En cuanto a la validación del caso clínico, no hubo diferencias significativas entre los dos grupos, profesores versus estudiantes. ( $\mathrm{p}>0.05)$. Todos los artículos recibieron una puntuación media superior a 2,75 y una puntuación media superior a 2,48. Conclusión: se concluye que el caso clínico fue elaborado y validado por las enfermeras especializadas y los estudiantes de enfermería de pregrado.

Palabras clave: Enfermería; Simulación; Educatión em enfermería.

\section{Introdução}

Os avanços tecnológicos e científicos têm reverberado na área da saúde, particularmente na área de enfermagem, por meio de informações que são produzidas em uma velocidade considerável, o que revela a importância das atualizações e a aquisição de metodologias de ensino que possam vir de encontro às mudanças da atualidade. Nesse aspecto, as metodologias ativas de ensino e de aprendizagem pretendem desenvolver o pensamento crítico e reflexivo, conectando o aluno às inovações científicas (Oliveira et al., 2014). Por isso, a educação continuada é um importante processo para o desenvolvimento, a atualização e o aprimoramento contínuo do conhecimento, quer seja para crescimento pessoal ou profissional (De Abreu, et al., 2014).

A preocupação com a formação profissional dos futuros enfermeiros tem sido objeto de diversos estudos (Oliveira et al., 2014: Johnsen, et al., 2016; Sarabia-Cobo, et al., 2016), os quais apontam para a importância do uso de ferramentas e de estratégias de ensino. Dentre essas ferramentas, destaca-se o uso da simulação que, por sua vez, vem alcançando reconhecimento e espaço nas universidades do mundo, particularmente nos cursos de graduação em enfermagem (Oliveira et al., 2014; Johnsen , et al., 2016).

Com a finalidade de instigar o raciocínio e o pensamento crítico, a simulação torna-se um recurso interessante, especialmente na enfermagem (Johnse, et al., 2016). Nesse contexto, a simulação realística é capaz de possibilitar a oportunidade de experimentar e de aprimorar habilidades práticas de forma prévia e em um local que tenha segurança, de maneira a minimizar agravos de saúde e riscos de acidentes para os alunos (Costa, et al., 2017). 
As tecnologias na área de saúde têm sido utilizadas para a condução dos alunos no desenvolvimento de controle motor, de decisão, inclusive de comunicação, usando um ambiente de simulação clínica virtual. Nesse sentido, a simulação clínica virtual é a recriação da realidade por meio de um sistema de computador, envolvendo pessoas reais que operacionalizam um sistema de simulação (Padilha, et al., 2019).

A simulação de paciente de alta fidelidade, quando comparada com outros métodos de ensino, revelou melhorar o conhecimento e o desempenho dos estudantes de enfermagem (La Cerra, et al., 2019), o que mostra a importância da elaboração de cenários e de casos clínicos que possam ser usados para melhorar a competência dos estudantes de enfermagem.

Assim, a simulação torna-se uma aliada no processo de ensino e aprendizagem em disciplinas que buscam desenvolver habilidades cognitivas e psicomotoras, como a disciplina de Sistematização da Assistência de Enfermagem. Dentre os conteúdos apresentados, o exame físico do sistema respiratório requer dos alunos diferentes saberes, o que muitas vezes é considerado pelo aluno como difícil e complexo. Assim, a simulação do exame físico do sistema respiratório cria um espaço propício à reflexão e à construção do saber. Assim, para que os estudantes desenvolvam imagens reais, é fundamental um caso clínico estruturado e capaz de explorar a compreensão do paciente e de seu estado de saúde.

Assim, como uma nova estratégia de ensino-aprendizagem, a simulação realística na área da saúde tem como proposta proporcionar situações reais através do uso de instrumentos norteadores como os casos clínicos, os manequins e os cenários realísticos (De Abreu, et al., 2015). A simulação apresenta as seguintes etapas: briefing, simulação, debriefing. Pode-se considerar o debriefing como a parte mais relevante da simulação. Além disso, um bom briefing e uma boa simulação são de grande importância para uma sessão de debriefing produtiva (Cuerva, et al., 2018). Dessa forma, o docente pode utilizar um caso clínico para compor um cenário com a finalidade de promover a aprendizagem experiencial a fím de facilitar a aquisição de habilidades psicomotoras e de raciocínio clínico dos estudantes. O uso de caso clínico para compor um cenário de simulação tem a capacidade de envolver os estudantes por meio da metodologia ativa e de aperfeiçoar sua habilidade de trabalhar a interdisciplinaridade e a conexão entre a teoria e a prática (Powers, 2020).

Na etapa denominada briefing ocorre a interação entre o facilitador e os estudantes, que apresenta o cenário, os objetivos e os objetivos de aprendizagem, pertinentes à experiência de simulação (Fabri, et al., 2017; Tyerman, et al., 2019)

Na simulação propriamente dita, é efetivada a participação dos estudantes na execução das ações propostas para o cenário de simulação (Nascimento,et al., 2020) e em seguida, tem-se o debriefing que corresponde a etapa final, marcada por um processo de diálogo e reflexão, que possibilita o desenvolvimento e a aquisição de habilidades clínicas dos participantes (Tyerman, et al., 2019).

Nesse aspecto, o presente estudo busca elaborar e validar um caso clínico para ser utilizado em simulação de uma situação que envolve um paciente com comprometimento do sistema respiratório. Esse caso clínico pretende ser uma ferramenta que irá auxiliar os docentes no processo ensino-aprendizagem na área de saúde, sendo que as pesquisas científicas mostram a utilização da simulação como um diferencial em relação a outras metodologias propostas em decorrência de estar assentada em reflexões contínuas e guiadas por um docente (Marmol, et al., 2012; Oliveira, et al., 2014).

Nesse contexto, o objetivo do presente estudo foi elaborar e validar um caso clínico para ser utilizado na simulação de uma situação realística do exame físico do sistema respiratório em um laboratório de práticas do Curso de Graduação em Enfermagem de uma Universidade Federal. 


\section{Metodologia}

Trata-se de um estudo metodológico do tipo aplicado, visando à construção e validação de um caso clínico para ser utilizado durante uma aula de simulação que tem como objetivo de aprendizagem ensinar os alunos a efetuarem o exame físico do sistema respiratório. A construção e a validação foram realizadas seguindo os passos da propedêutica descritos por Barros, et al., (1996) e Jarvis (2002). Buscou-se conhecimento cientifico existente sobre o assunto por meio de uma revisão de literatura realizada no Portal de Periódicos da Capes, utilizando-se três descritores: 'simulação', 'aprendizagem' e 'educação em enfermagem' e, foi utilizado o operador boleano AND. Os critérios de inclusão foram artigos publicados entre os anos 2014 e 2019, estes, escritos em inglês, português e espanhol e que respondessem a questão norteadora: "Como a simulação tem sido utilizada no ensino da enfermagem?”. Como critérios de exclusão, os estudos que não puderam ser obtidos na íntegra. Selecionou-se 10 artigos que serviram como subsídio para a elaboração do caso clínico. Após a revisão de literatura, o caso clínico elaborado constou dos seguintes itens:

Dados gerais: idade, sexo, profissão, raça, história prévia, admissão e queixa principal; Dados Clínicos: dados de anamnese; Dados terapêuticos: dados relacionados com a terapêutica; Exame físico respiratório: inspeção estática; inspeção dinâmica; palpação; percussão e ausculta; Diagnóstico de enfermagem, no qual, foram elaborados dois: Padrão respiratório ineficaz relacionado à fadiga da musculatura respiratória evidenciado por ortopneia, padrão respiratório anormal; Troca de gases prejudicada relacionada à desequilíbrio na relação ventilação-perfusão evidenciado por respiração anormal (ex: frequência, ritmo, profundidade) seguindo a taxonomia da NANDA (Herdman \& Kamitsuru, 2017).

Uma vez elaborado, o caso clínico foi submetido à validação de conteúdo. A validação foi realizada em duas etapas; a primeira, por docentes do curso de graduação em Enfermagem e a segunda, por alunos da graduação em Enfermagem. Na primeira etapa, uma versão prévia do caso clínico foi submetida à avaliação por cinco docentes com experiência na área de semiologia e de semiotécnica, com no mínimo graduação em enfermagem com título de especialista e que concordaram em participar da pesquisa, assinando o termo de consentimento livre e esclarecido (TCLE). Foi solicitado aos docentes a leitura do caso clínico e posteriormente sugestões para modificar os seguintes itens que compõem o estudo de caso: dados gerais; dados clínicos; dados terapêuticos; exame físico e diagnósticos de enfermagem. Também questionaram- se a idade, gênero, formação, tempo de formação e titulação acadêmica de cada docente participante.

O instrumento utilizado pelo Comitê de Juízes foi uma escala de quatro pontos, na qual se avaliaram os itens: dados gerais, dados clínicos e dados terapêuticos conforme a clareza, a compreensão a linguagem utilizada e a relevância, sendo a pontuação zero (0) caracterizada como não adequado; um (1), adequado com várias alterações; dois (2), adequado com poucas alterações e três (3), como adequado sem alterações. Se o profissional pontuasse o caso clínico na escala com a pontuação 0,1 ou 2, este teria que sugerir as modificações necessárias.

Cada item foi avaliado e as modificações sugeridas foram realizadas, sendo considerado adequado quando todos os itens do caso clínico alcançassem uma pontuação acima de três (3) pontos, conforme a escala.

Concomitantemente à primeira etapa, o caso clínico também foi avaliado por alunos do curso de graduação em enfermagem, cuja amostra de conveniência foi de cinco (5) alunos. Como critérios de inclusão, os alunos deveriam estar devidamente matriculados no curso de graduação em enfermagem da Universidade Federal de Uberlândia, a partir do terceiro período do curso e concordar em participar da pesquisa, mediante o aceite do TCLE. Foram excluídos os alunos que estavam no primeiro e no segundo período do Curso de Enfermagem. Foi utilizado o mesmo instrumento, além disso, os alunos deveriam avaliar os mesmos tópicos que os docentes avaliaram. Os alunos foram abordados pessoalmente pela pesquisadora principal, quando foram explicados os objetivos do estudo. 
Posteriormente, foram entregues o caso clínico e o instrumento de avaliação. Os alunos tiveram um prazo de aproximadamente uma semana para devolver o caso clínico com as devidas considerações. Os pesquisadores efetuaram todas as correções solicitadas pelo Comitê de juízes e pelos alunos.

A caracterização da amostra dos docentes e alunos que participaram na validação foi analisada de forma descritiva, sendo que os dados foram apresentados como média, mediana e desvio padrão. A normalidade dos dados, por grupo (docente ou discente), foi testada pelo teste de Shapiro-Wilk; e pelo menos um dos grupos não apresentou distribuição Gaussiana. Sendo assim, os grupos foram comparados pelo teste de Mann-Whitney para dados não pareados. Adotou-se significância de 0.05 para todos os testes. O projeto de pesquisa foi encaminhado para a apreciação do Comitê de Ética em Pesquisa da Universidade Federal de Uberlândia e aprovado sob parecer $n^{\circ} 1.864 .940$.

\section{Resultados}

Os avaliadores foram predominantemente do sexo feminino nos dois grupos, sendo cinco mulheres no grupo dos docentes e quatro mulheres e um homem no grupo dos discentes. As idades foram diferentes nos dois grupos $(\mathrm{p}=0,008)$, sendo que os docentes apresentaram idade mediana superior aos alunos (38 versus 23 anos).

Quanto aos itens da validação do caso clínico, nenhum item apresentou diferença significativa entre os dois grupos, docentes versus discentes $(\mathrm{p}>0,05)$. Todos os itens receberam avaliação mediana superior a 2,75 e média, superior a 2,48. O caso clínico foi avaliado como adequado tanto pelos docentes como pelos discentes, recebendo notas, em sua maioria, próximas de três (limite da escala Likert) (Tabela 1).

Tabela 1 - Idade e Escores médios, medianos e variação para os itens de validação do caso clínico $(\mathrm{n}=$ 5 cada grupo).

\begin{tabular}{|c|c|c|c|c|}
\hline \multirow[b]{3}{*}{ Item } & \multicolumn{2}{|c|}{ Grupo } & \multirow[b]{3}{*}{$p^{1}$} & \multirow[b]{2}{*}{ Todos } \\
\hline & Docentes & Discentes & & \\
\hline & Média (Mediana) & Média (Mediana) & & $\begin{array}{c}\text { Média } \pm \text { Desvio Padrão } \\
\text { (Mediana) }\end{array}$ \\
\hline Idade & $38.20(38.00)$ & $23.00(23.00)$ & 0.008 & $30.60 \pm 9.90(26.00)$ \\
\hline A & $2.85(3.00)$ & $2.70(2.50)$ & 0.421 & $2.78 \pm 0.25(2.88)$ \\
\hline B & $2.40(2.75)$ & $2.55(3.00)$ & 0.690 & $2.48 \pm 0.72(2.88)$ \\
\hline $\mathrm{C}$ & $2.95(3.00)$ & $2.85(3.00)$ & 0.548 & $2.90 \pm 0.17(3.00)$ \\
\hline $\mathrm{D}$ & $2.65(3.00)$ & $2.60(2.50)$ & 0.841 & $2.63 \pm 0.43(2.75)$ \\
\hline $\mathrm{E}$ & $2.40(3.00)$ & $3.00(3.00)$ & 0.690 & $2.70 \pm 0.95(3.00)$ \\
\hline
\end{tabular}

${ }^{1}$ Probabilidade baseada no teste de Mann-Whitney, para comparação entre docentes e discentes.

Fonte: Autores (2019).

Os avaliadores realizaram modificações importantes no conteúdo do material, sendo que o tópico "Dados Clínicos" foi o único que recebeu tais modificações. Os avaliadores concederam como recomendação principal a ordem dos acontecimentos, uma vez que os avaliadores apontaram que tiveram dificuldade de compreensão dos fatos e solicitaram que estes fatos deveriam acontecer de forma cronológica. Outra sugestão feita foi em relação à troca de nome do item "dados clínicos" para "anamnese", uma vez que se entende que os dados clínicos são componentes da anamnese realizada pelo enfermeiro. Os pesquisadores acataram as recomendações feitas pelos especialistas e, com isso, o caso clínico foi validado pelos docentes após correções realizadas de acordo com as sugestões dadas conforme mostra a Figura 1. 
Figura 1. Caso clínico após modificações sugeridas pelos avaliadores. Uberlândia - MG, 2021.

\begin{tabular}{|l|}
\hline $\begin{array}{c}\text { CASO CLÍNICO - SISTEMA RESPIRATÓRIO } \\
\text { DADOS GERAIS }\end{array}$ \\
\hline $\begin{array}{l}\text { J.H, } 38 \text { anos, guarda de trânsito, casado, sem filhos, branco, com história prévia de boa saúde, sem doença crônica, sem antecedentes familiares. } \\
\text { Admitido na unidade de saúde no dia 13/01/2017. Queixa Principal: Dispnéia e fadiga. }\end{array}$ \\
\hline \multicolumn{1}{c|}{ ANAMNESE } \\
\hline $\begin{array}{l}\text { Durante a anamnese, o senhor J.H. relata ser tabagista e que vem apresentando episódios de dispnéia e fadiga ao caminhar por pequenas } \\
\text { distâncias, além de tosse no início da manhã há cerca de } 10 \text { anos. Em agosto de 2016, apresentou "resfriado", acompanhado de tosse estridente } \\
\text { grave e secreção esbranquiçada, espessa, em média quantidade. Nos últimos três meses, teve episódios de dispnéia mais freqüentes, mesmo em } \\
\text { repouso. Refere ortopneia, sendo necessária a utilização de dois travesseiros quando em repouso no leito; acorda cerca de três vezes durante a } \\
\text { noite. No momento, apresenta produção de escarro branco e espumoso, em pequena quantidade e relata dor branda medioesternal, intensidade 2. } \\
\text { Refere precisar de auxilio, pois é incapaz de realizar percursos pequenos, sem apresentar quadros de fadiga. }\end{array}$ \\
\hline
\end{tabular}

\section{DADOS TERAPÊUTICOS}

Não realizado tratamento medicamentoso até o momento. Relata ausência de alergias, hospitalizações ou lesões do tórax. Sem antecedentes familiares para tuberculose, asma ou câncer. Relata ser tabagista há 30 anos e fazer o uso de bebidas alcoólicas há 12 anos. Refere consumir, aproximadamente, seis latas por semana. Ausência de hemoptise, sudorese noturna ou dispnéia paroxística noturna.

\section{EXAME FÍSICO}

Inspeção estática: Não foi notada presença de cicatrizes, abaulamentos ou retrações. Tórax simétrico, coloração normal. Mamas sem alterações. Inspeção dinâmica: Freqüência respiratória em repouso de $24 \mathrm{irpm} / \mathrm{min}$, regular e profunda, com expiração prolongada. Freqüência respiratória em movimento de $34 \mathrm{irpm} / \mathrm{min}$. Expansibilidade torácica simétrica nos dois hemitórax, apesar de diminuída. Faz uso de musculatura acessória. Palpação: Expansão torácica diminuída, mas simétrica. Frêmito toracovocal simetricamente diminuído bilateralmente. Ausência de nódulo, massas ou sensibilidade à palpação.

Percussão: Presença de excursão diafragmática de $1 \mathrm{~cm}$ e simétrica bilateralmente. Som claro pulmonar em todos os campos pulmonares. Ausculta: Presença de murmúrio vesicular diminuído, na porção anterior esquerda. Sibilo expiratório em todo o tórax posterior, sendo menor em hemitórax direito.

\section{DIAGNÓSTICO DE ENFERMAGEM (NANDA 2015-2017)}

Padrão respiratório ineficaz relacionado à fadiga da musculatura respiratória evidenciado por ortopnéia, padrão respiratório anormal. Troca de gases prejudicada relacionado à desequilíbrio na relação ventilação-perfusão evidenciado por respiração anormal (ex: freqüência, ritmo, profundidade.)

Fonte: Autores.

\section{Discussão}

A elaboração e a validação de um caso clínico são essenciais para que a simulação seja clara e coerente com o objetivo de estimular o raciocínio clínico e as habilidades psicomotoras. No presente estudo, a validação de um caso clínico que estimula o processo ensino-aprendizagem do exame físico do sistema respiratório pode ser útil na aprendizagem ativa e na promoção da autoconfiança dos estudantes. A aprendizagem ativa contribui tanto para a satisfação do estudante com relação à simulação quanto para a autoconfiança no gerenciamento da situação do paciente simulado, o que mostra que a aprendizagem é construída em ambientes que possibilitem a interação dos estudantes (Olaussen, et al., 2020).

A utilização de simulação e de metodologias ativas no processo de formação dos estudantes de enfermagem viabiliza a forma de pensar em relação ao processo de aprendizagem sob uma perspectiva de construção de saberes em que professores e estudantes se envolvem verdadeiramente (David, et al., 2019). Resultado semelhante foi identificado em outro estudo, o qual mostra que o uso da simulação é importante, pois estimula o raciocínio clínico, além de estimular a autoconfiança do aluno (Ferreira, et al., 2018).

Para tanto, o presente caso clínico foi elaborado com base na literatura e se procurou trazer informações relevantes e necessárias sobre o procedimento. Posteriormente, o caso foi validado por docentes que trabalham com o fenômeno do estudo e por alunos que identificaram as dificuldades quanto à compreensão e à linguagem utilizada. Estudo cujo objetivo foi validar um cenário, também destaca a importância de cenários clínicos realísticos e que apresentem objetivos claros e que permitam ao 
aluno e ao profissional de enfermagem vivenciar a prática (Garbuio, et al., 2016). Nesse aspecto, o caso clínico passa a ser fundamental para a elaboração de um cenário que torne o ambiente seguro e se aproxime ao máximo da realidade clínica, de modo a proporcionar satisfação aos estudantes e aos profissionais.

O caso clínico utilizado na simulação em vídeo, particularmente em momentos como o da pandemia, devido ao fechamento de universidades, mostra-se como uma opção de ensino de simulação de alta fidelidade. Estudo mostra que consultas de vídeo simuladas possibilitam o treinamento na modalidade de atenção à saúde para enfrentar os desafios emergentes nos serviços de saúde (Jiménez-Rodríguez, \& Arrogante, 2020).

Os avaliadores realizaram modificações importantes no conteúdo do material. O único tópico que teve sugestões foi o item "Dados Clínicos". A principal recomendação feita foi em relação à ordem dos acontecimentos, uma vez que os avaliadores apontaram que tiveram dificuldade de compreensão dos fatos e solicitaram que estes fatos deveriam acontecer de forma cronológica. Outra sugestão feita foi em relação à troca de nome do item "dados clínicos" para "anamnese", uma vez que, entende-se que os dados clínicos são componentes da anamnese realizada pelo enfermeiro. Com isso, o caso clínico foi validado pelos docentes após correções realizadas de acordo com as sugestões dadas.

No que diz respeito à caracterização dos docentes, houve prevalência do sexo feminino, o que corrobora a característica da profissão, a qual é exercida predominantemente por mulheres. Todas tinham ao menos a especialização, o que contribui para a obtenção de conhecimento científico, somado à experiência clínica.

A utilização de um instrumento para avaliação de um caso clínico pelos docentes permitiu organizar as informações de forma coesa e uniforme, tornando uma discussão construtiva e relevante para a adequação do caso clínico e, por fim, para a validação do instrumento.

Quanto à avaliação feita pelos alunos, observou-se que compreenderam o caso clínico como um todo e cada uma das suas partes e validaram o instrumento. Este resultado mostra que o caso clínico é de fácil compreensão, já que a maior parte dos alunos estava no terceiro período do curso de graduação em enfermagem. Com isso, enfatiza-se a importância de adequar o conteúdo ao nível educacional e cultural do paciente, com o intuito de evitar a limitação de aprendizado em decorrência da baixa escolaridade, uma vez que o aluno passa a ser mais crítico e observador ao longo da graduação.

Por isso, os materiais educativos devem ser escritos de forma simples, clara e objetiva, de modo a permitir a transmissão de informações precisas. Assim, a participação de especialistas no assunto e de indivíduos que receberão as informações aumenta a credibilidade e melhora do conteúdo do manual informativo, tornando-o com uma linguagem acessível (Silva Maciel, et al. 2016). Dessa forma, o caso clínico foi redigido de forma a ser compreendido tanto pelo especialista quanto pelos alunos que estão em processo de formação, para que possam atuar no cenário de simulação de maneira segura e real.

Estudo que buscou informar estratégias educacionais futuras, sintetizando pesquisas relacionadas a recursos de aprendizagem combinada, usando vídeos de simulação para ensinar habilidades clínicas para estudantes de saúde, mostrou que os vídeos de simulação podem ser uma ferramenta útil para ensinar habilidades clínicas para estudantes de saúde, incluindo enfermagem (Coyne, et al., 2018). Nesse sentido, a validação do caso clínico para ser usado em vídeo aponta possibilidades para o ensino remoto, em uma sala de aula virtual, com resultados promissores.

Uma metanálise trouxe resultados que sugerem que as intervenções educacionais de enfermagem fundamentadas em simulação apresentam fortes efeitos educacionais, com efeitos particularmente grandes no domínio psicomotor, sendo que o efeito não foi proporcional ao nível de fidelidade (Kim, et al., 2016). Usar uma variedade de intervenções educacionais para responder a todos os objetivos educacionais é extremamente relevante (Kim, et al., 2016). Nesse caso, o presente estudo, ao validar um caso clínico de fácil compreensão, tanto por parte dos alunos como dos docentes, cumpre esse papel de atender à multiplicidade de objetivos educacionais, superando, assim, o desafio de incorporar atividades teórico-práticas que visam superar a fragmentação herdada do ensino tradicional (Fonseca, et al., 2020). 
Nessa vertente, a simulação é uma ferramenta de ensino-aprendizagem cujas evidências científicas apontam o potencial para a interdisciplinaridade e para o desenvolvimento de competências e de habilidades nos diversos ramos do saber (Fonseca, et al., 2020). Isso mostra a importância da validação de ferramentas que possam fomentar a articulação entre os saberes. O caso clínico validado envolve o exame físico respiratório, tema, em geral, presente nos planos de ensino de diversas disciplinas da área de enfermagem.

\section{Conclusão}

O caso clínico foi elaborado e validado pelos enfermeiros especialistas e pelos alunos do Curso de Graduação em Enfermagem, com poucas alterações que foram efetuadas, sendo considerado adequado para ser utilizado no ensino por meio da simulação do exame físico do sistema respiratório. O caso clínico poderá ser utilizado por diversas instituições de graduação em enfermagem, com o intuito de instruí-los sobre a execução precisa do exame físico do sistema respiratório, além de estimular o raciocínio clínico e a habilidade para desenvolver o processo de enfermagem.

\section{Referências}

Abreu, A. G., de Freitas, J. S., Berte, M., Ogradowski, K. R. P., \& Nestor, A. (2014). O uso da simulação realística como metodologia de ensino e aprendizagem para as equipes de enfermagem de um hospital infanto-juvenil: relato de experiência. Ciência \& Saúde, 7(3), 162-166.

Barros, A. L. B. L. D., Glashan, R. D. Q., \& Michel, J. L. M. (1996). Bases propedêuticas para a prática de enfermagem-uma necessidade atual. Acta Paul Enferm., 9(1), 28-37.

Coyne, E., Rands, H., Frommolt, V., Kain, V., Plugge, M., \& Mitchell, M. (2018). Investigation of blended learning video resources to teach health students clinical skills: an integrative review. Nurse education today, 63, 101-107

Cuerva, M. J., Piñel, C. S., Martin, L., Espinosa, J. A., Corral, O. J., \& Mendoza, N. (2018). Teaching childbirth with high-fidelity simulation. Is it better observing the scenario during the briefing session? Journal of Obstetrics and Gynaecology, 38(5), 607-610.

David, F. S., dos Santos, M. D. S. S., Braga, A. C. G., de Almeida Guilherme, F. J., \& de Mendonça Henrique, D. (2018). Evolution for emancipatory praxis: development of the realistic simulation method in undergraduate teaching in nursing. Online Brazilian Journal of Nursing, $17(1), 13$.

Fabri, R. P., Mazzo, A., Martins, J. C. A., Fonseca, A. D. S., Pedersoli, C. E., Miranda, F. B. G., ... \& Baptista, R. C. N. (2017). Development of a theoreticalpractical script for clinical simulation. Revista da Escola de Enfermagem da USP, 51.

Ferreira, R. P. N., Guedes, H. M., Oliveira, D. W. D., \& de Miranda, J. L. (2018). Simulação realística como método de ensino no aprendizado de estudantes da área da saúde. Revista de Enfermagem do Centro-Oeste Mineiro, 8.

Fonseca, L. M. M., Monteiro, J. C. D. S., Aredes, N. D. A., Bueno, J. V., Domingues, A. N., Coutinho, V. R. D., \& Baptista, R. C. N. (2020). Cenário de simulação interdisciplinar na educação em enfermagem: parto e nascimento humanizados. Revista Latino-Americana de Enfermagem, 28.

Garbuio, D. C., Oliveira, A. D. S., Kameo, S. Y., Melo, E. S., Dalri, M. C. B., \& Carvalho, E. C. (2016). Simulação clínica em enfermagem: relato de experiência sobre a construção de um cenário. Rev Enferm UFPE, 10(8), 3149-55.

Herdman TH, Kamitsuru S. NANDA International nursing diagnoses: Definitions classification, 2018-2020. New York: Theime; 2017. http://dx.doi.org/10.1055/b-006-161141

Jarvis, C. (2002). Exame físico e avaliação de saúde. Rio de Janeiro, 14, 455-456.

Jiménez-Rodríguez, D., \& Arrogante, O. (2020). Simulated video consultations as a learning tool in undergraduate nursing: Students' perceptions. In Healthcare, 8 (3), 280.

Johnsen, H. M., Fossum, M., Vivekananda-Schmidt, P., Fruhling, A., \& Slettebø, Å. (2016). Teaching clinical reasoning and decision-making skills to nursing students: Design, development, and usability evaluation of a serious game. International journal of medical informatics, 94, 39-48.

Kim, J., Park, J. H., \& Shin, S. (2016). Effectiveness of simulation-based nursing education depending on fidelity: a meta-analysis. BMC medical education, 16(1), 1-8.

La Cerra, C., Dante, A., Caponnetto, V., Franconi, I., Gaxhja, E., Petrucci, C., ... \& Lancia, L. (2019). Effects of high-fidelity simulation based on lifethreatening clinical condition scenarios on learning outcomes of undergraduate and postgraduate nursing students: a systematic review and meta-analysis. $B M J$ open, $9(2)$, e025306.

Maciel, B. S., Barros, A. L. B. L. D., \& Lopes, J. D. L. (2016). Elaboração e validação de um manual informativo sobre cateterismo cardíaco. Acta Paulista de Enfermagem, 29(6), 633-642. 
Research, Society and Development, v. 10, n. 6, e48310616023, 2021

(CC BY 4.0) | ISSN 2525-3409 | DOI: http://dx.doi.org/10.33448/rsd-v10i6.16023

Marmol, M. T., Braga, F. T. M. M., Garbin, L. M., Moreli, L., Santos, C. B. D., \& Carvalho, E. C. D. (2012). Central catheter dressing in a simulator: the effects of tutor's assistance or self-learning tutorial. Revista latino-americana de enfermagem, 20(6), 1134-1141.

Nascimento, J. D. S. G., Costa, A. B. F., Sangiovani, J. C., dos Santos Silva, T. C., Regino, D. D. S. G., \& Dalri, M. C. B. (2020). Pré-simulação, pré-briefing ou briefing na simulação em enfermagem: quais as diferenças? Revista Eletrônica de Enfermagem, 22.

Olaussen, C., Heggdal, K., \& Tvedt, C. R. (2020). Elements in scenario-based simulation associated with nursing students' self-confidence and satisfaction: A cross-sectional study. Nursing open, 7(1), 170-179.

Oliveira Costa, R. R., de Medeiros, S. M., Martins, J. C. A., Cossi, M. S., \& de Araújo, M. S. (2017). Percepção de estudantes da graduação em enfermagem sobre a simulação realística. Revista Cuidarte, 8(3), 1799-1808.

Oliveira, S. N. D., Prado, M. L. D., \& Kempfer, S. S. (2014). Utilização da simulação no ensino da enfermagem: revisão integrativa. Revista Mineira de Enfermagem, 18(2), 487-504.

Padilha, J. M., Machado, P. P., Ribeiro, A., Ramos, J., \& Costa, P. (2019). Clinical virtual simulation in nursing education: randomized controlled trial. Journal of medical Internet research, 21(3), e11529.

Powers, K. (2020). Bringing simulation to the classroom using an unfolding video patient scenario: A quasi-experimental study to examine student satisfaction, self-confidence, and perceptions of simulation design. Nurse education today, 86, 104324.

Sarabia-Cobo, C. M., Alconero-Camarero, A. R., Lavín-Alconero, L., \& Ibáñez-Rementería, I. (2016). Assessment of a learning intervention in palliative care based on clinical simulations for nursing students. Nurse education today, 45, 219-224.

Tyerman, J., Luctkar-Flude, M., Graham, L., Coffey, S., \& Olsen-Lynch, E. (2019). A systematic review of health care presimulation preparation and briefing effectiveness. Clinical Simulation in Nursing, 27, 12-25. 